\title{
Euro area inflation expectations
}

\author{
Bicardo Gimeno and Eva Ortega
}

This study explores the recent dynamics of inflation expectations for the main euro area countries. It uses daily financial data for the main euro area countries over the past 15 years with a wide range of time horizons. The estimation of a model of the term structure of inflation expectations using these data allows the common part to be separated from the countryspecific part. It is found that, for the various time horizons and countries, the bulk of expected inflation is common to the whole euro area. The weight of country-specific factors is low, being most significant for the shorter term. For time horizons between five and ten years, the estimated inflation expectations showed a downward trend from 2012, which has reversed in the last two years owing to the application of a broad set of unconventional monetary policy measures in the euro area since mid-2014. Still, in the past year, medium-term inflation expectations have held below $2 \%$, around $1.7 \%$ on average, clearly lower than in the period before the economic crisis. 


\section{EURO AREA INFLATION EXPECTATIONS}

The authors of this article are Ricardo Gimeno and Eva Ortega, of the Associate Directorate General Economics and Research. ${ }^{1}$

Introduction

\section{Measurement of inflation expectations for the various countries and terms}

How inflation expectations are formed in countries which share monetary policy is not a trivial question. Inflation expectations are a key indicator for monetary policy, since they reflect the private sector's perception of future inflation developments and the public's confidence in the central bank's ability to achieve its price stability mandate. In the long term and with a common monetary authority enjoying full credibility, the economic agents of all the countries in a monetary union should expect the same inflation rate in each of their economies: the rate constituting the monetary authority's target. However, as shown by Chart 1, agents' expectations as to the future course of inflation at shorter time horizons may differ from one country to another as a result of specific factors affecting their inflation dynamics. ${ }^{2}$

We estimate the behaviour of inflation expectations in euro area countries using daily financial market data and an empirical model of the term structure of expected inflation. Among other things, we look at whether the common behaviour predominates in all years of the analysis period and in all time horizons, or whether divergences between countries are apparent. The methodology employed also allows us to shed light on whether inflation expectations have been firmly anchored to the monetary policy target.

The accurate measurement of inflation expectations is complex in view of its unobservable nature. The information for estimating them is usually obtained from two alternative sources: surveys conducted by economic agents and information drawn from financial markets. For the individual euro area countries, the most complete survey on inflation expectations is Consensus Forecast, which puts monthly questions to consumers and firms on, among other variables, the expected annual inflation in a wide selection of countries for the current year and the next. Also, twice a year the time horizon is lengthened to ten years. ${ }^{3}$ This source, despite providing a genuine measure of expectations, contains information for a limited number of time horizons and is available only once a month with a two-week lag.

In contrast to these limitations, the financial markets provide abundant daily real-time information on inflation expectations. First, there are indexed sovereign bonds which provide protection against future inflation. In the euro area, although these bonds are issued by national authorities, most are indexed to the inflation of the euro area as a whole and thus do not provide information on the expected inflation in each country. Moreover, they are scantly comparable across countries owing to their different liquidities and risk levels. Second, traded daily on the markets are inflation swaps linked to the inflation of the euro area and of its main countries (France, Italy, Spain and Germany), with 15 maturities ranging from 1 to 30 years, which contain information on the expected future course of

1 This article updates the analysis and summarises the main ideas and results reported in Gimeno and Ortega (2016).

2 These may include, for example: differences in dependence on oil, such that the same change in world oil prices has different impacts on short-term inflation in different countries; or fiscal measures adopted by a specific country which affect its indirect taxes or its regulated prices; or different wage dynamics reflected in different pressures on prices.

3 The European Commission's Business and Consumers Survey enquires about expected inflation only for time horizons of one or two years. 


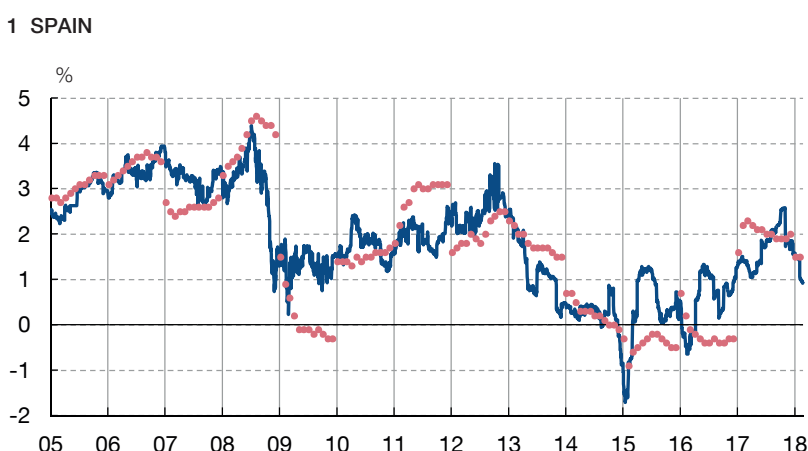

2 ITALY

3 FRANCE

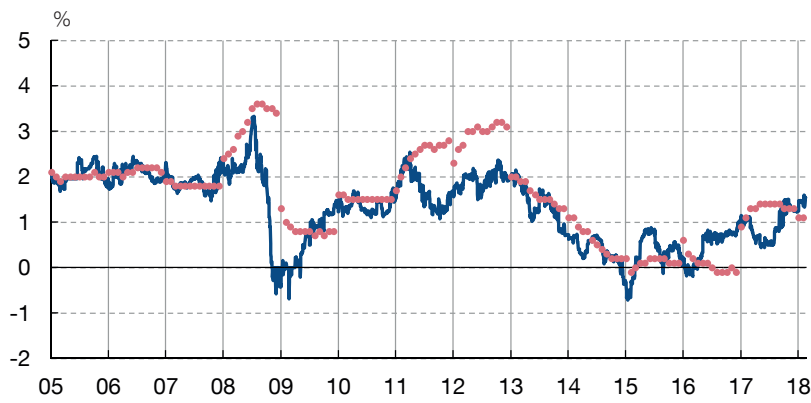

4 GERMANY
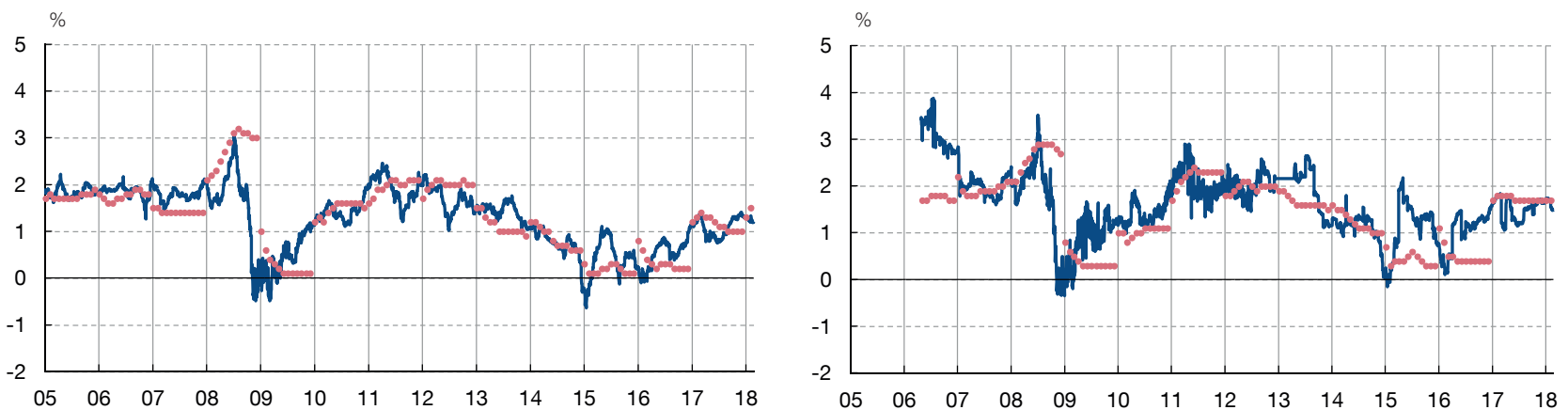

INFLATION SWAPS

- CONSENSUS FORECAST SURVEY

SOURCES: Banco de España and Consensus Forecast.

inflation at different time horizons for various euro area economies. These swaps are contracts in which one counterparty exchanges a consideration linked to inflation during the term of the contract in exchange for a fixed rate ("inflation compensation") which includes both expected average inflation for that term and possible risk premia. ${ }^{4}$

Chart 1 compares the expected year-on-year inflation for the aforementioned four countries for the current year reported monthly by Consensus Forecast with the average inflation compensation in one-year inflation swap contracts (whose reference inflation is the observed year-on-year rate three months before contract maturity ${ }^{5}$ ). It shows that the inflation compensation on swaps fluctuates more than the expectations indicated in the survey, reflecting a greater ability to react to any new information received by agents, although both time series exhibit very similar trends over time. ${ }^{6}$ This same result is obtained if the survey and swaps are compared at longer terms (see Gimeno and Ortega, 2016).

4 For more information on the various financial assets linked to inflation, see Fuertes and Gimeno (2017) and Gimeno and Ibáñez (2017).

5 Consensus Forecast makes year-end predictions, whereas in swaps the maturity is measured from the trade date (it is therefore a moving reference). As a result, the overlap between these two indicators varies throughout the year and they only overlap fully in April each year, since the overlap decreases with the time separation with respect to the previous April and to the coming April. In the case of swaps, the value shown is the mean of the prices published by different contributors.

6 Gimeno and Ortega (2016) also explore whether the greater volatility of the measure of inflation compensation on swaps may be partly due to their low liquidity on some inflation swap markets, which would be reflected in a wide spread between the highest and lowest compensations traded (known as the bid-ask spread). However, except for shorter time horizons in the period 2007-2012, the spreads are not wide, do not fluctuate greatly and do not differ markedly between countries. The inflation swap market is more liquid (lower spreads) at higher terms, where inflation protection may be more important. 
Do expected inflation rates exhibit common behaviour at the various time horizons in the euro area countries?
To answer this question, we estimated a model of the term structure of expected inflation based on the model of interest rate term structure of Diebold, Li and Yue (2008), in turn inspired on Nelson and Siegel (1987). Our estimate uses jointly the daily inflation compensation time series of inflation swap contracts for Germany, France, Italy, Spain and the aggregate euro area, at all available terms from 1 to 30 years. ${ }^{7}$ The sample begins on 30 June $2004^{8}$ and ends on 20 February 2018. It is a factor model, which, since it extracts common components, eliminates a good part of the measurement noise usually found in high-frequency financial data. Additionally, since it uses jointly the information of various countries, the model enables us to separate the common and country-specific portions of the expected behaviour of inflation for different time horizons.

The results of the estimation suggest that, for the various time horizons and countries, the bulk of inflation compensation is common for the whole euro area. The weight of the specific factors of one country or another is small, being more significant for shorter terms.

Specifically, we estimate the three factors characterising the term structure of inflation compensation: the long-term level, the slope or difference between long and short term, and the curvature or transition from the short to the long term. Each of these three factors is broken down into a common portion, identified using the information from inflation swaps for the total euro area, and a national or country-specific portion. Chart 2 shows the time behaviour of the estimated factors. The panels in the left-hand column separate the component common to the whole euro area from the country-specific components, while the panels in the right-hand column represent the level, slope and curvature for each country as an aggregation of its common and specific components.

As expected in a monetary union, the common component predominates in long-term expected inflation in the euro area over the whole period analysed (top panels of Chart 2). Although there are discrepancies between countries some periods, these are minor. It is also notable how the common behaviour of long-term trend inflation compensation fluctuates over time. Thus, after a sharp fall in the 2009 global crisis, it subsequently recovered very rapidly. At the end of 2012 it began another bout of decreases, this time much more gradual, which gave rise to certain concern over possible deanchoring of longterm inflation expectations in the euro area and contributed to the announcement and implementation of further unconventional monetary policy measures by the ECB from mid2014, including the Asset Purchase Programme, to guide inflation towards its objective of below, but close to, 2\% (see Draghi, 2014, 2016 and 2017). These measures managed to slow the downward trend of long-term expected inflation, which finally recovered at the end of 2016 to levels close to those before the crisis and has remained steady at those levels since then.

The middle and bottom left-hand panels of Chart 2 show that the factors governing the shorter time horizons of the inflation compensation curve (the slope or difference between long-term and short-term expected inflation, and the curvature or transition from short to long term) are less clearly dominated by the common factor. The country-specific components are as large as the common one and the differences between countries may

7 Also estimated is a liquidity risk compensation, which is identified by using in the model estimation the time series of the bid-ask spreads for the same countries and terms. As illustrated by Chart 3 , the liquidity premium is very small and does not influence the results. See Gimeno and Ortega (2016) for details of the model identification and estimation.

8 For Germany, the sample begins in April 2006. 
1 LONG-TERM LEVEL

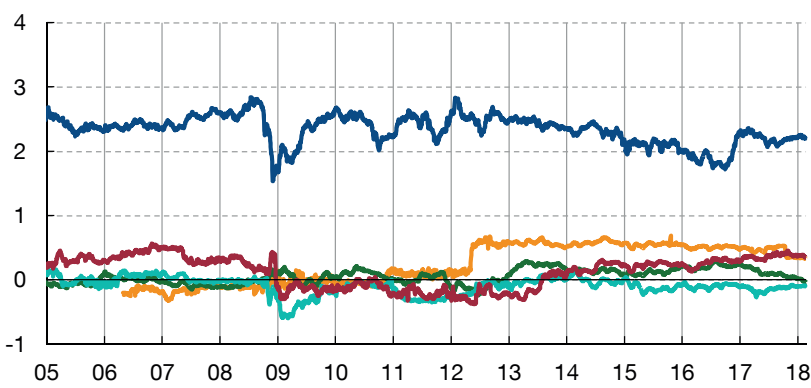

GERMANY FRANCE ITALY

2 DIFFERENCE BETWEEN LONG AND SHORT TERM

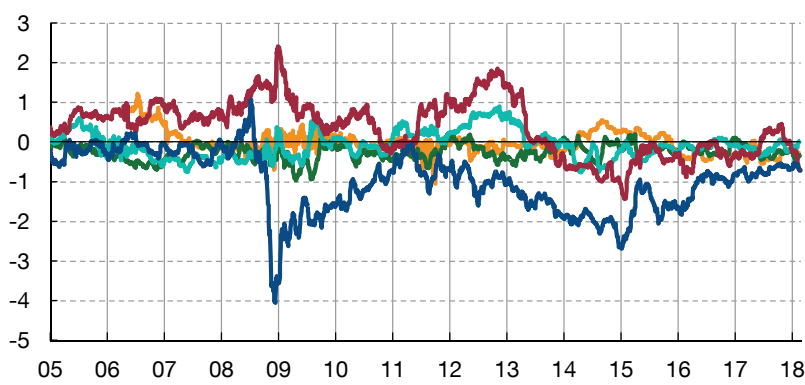

$\begin{array}{ll}\text { GERMANY } & \text { FRANCE } \\ \text { ITALY } \\ \text { COMMON, EURO AREA }\end{array}$

3 CURVATURE (TRANSITION FROM SHORT TO LONG TERM)

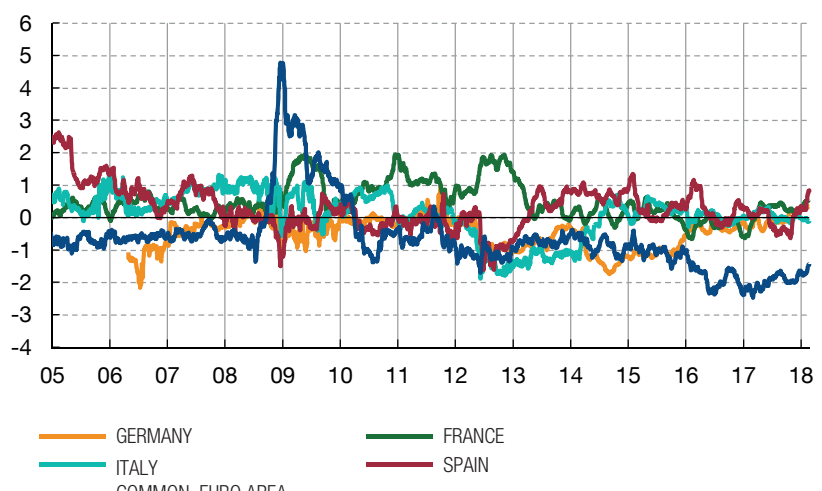

1 LONG-TERM LEVEL

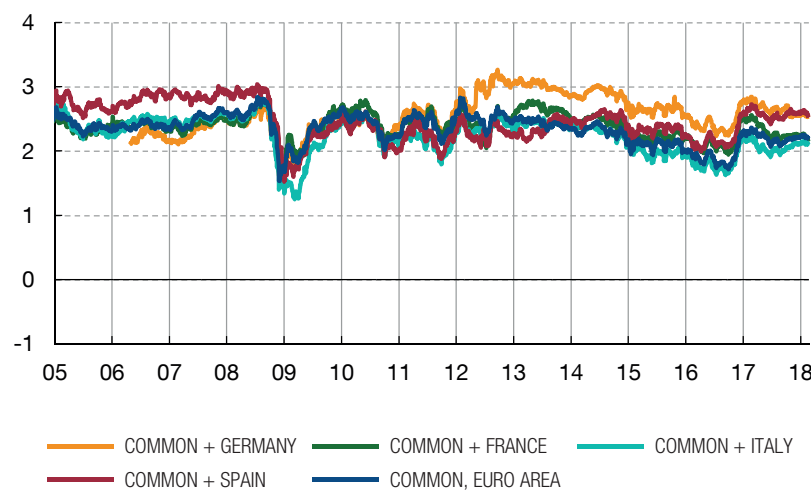

2 DIFFERENCE BETWEEN LONG AND SHORT TERM

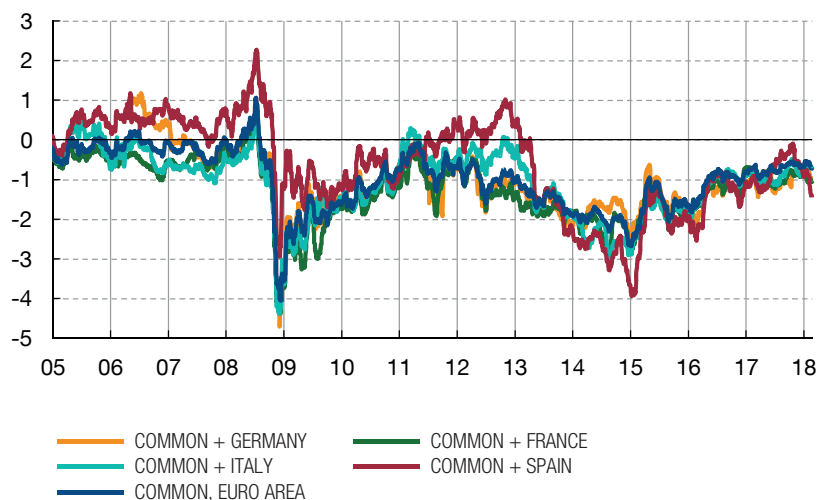

3 CURVATURE (TRANSITION FROM SHORT TO LONG TERM)

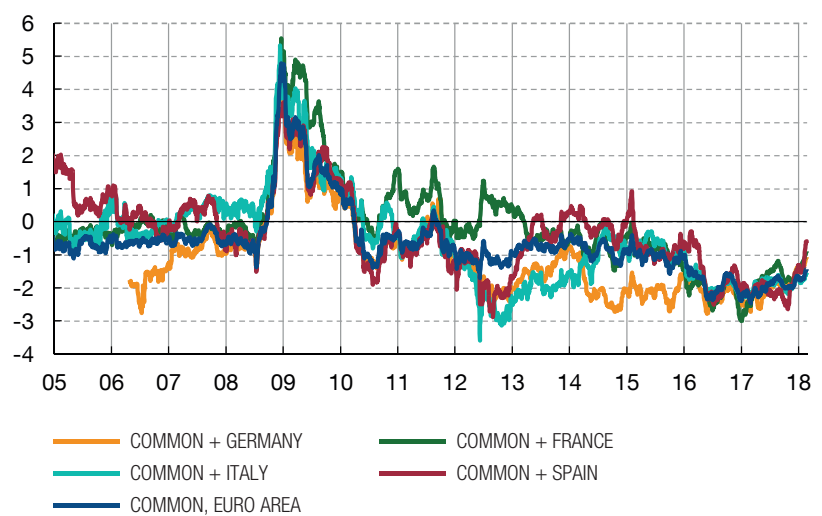

SOURCE: Banco de España.

a Factors obtained from the model of Gimeno and Ortega (2016), which estimates the term structure of inflation compensation using daily financial data for expected inflation at terms of 1 to 30 years. There dynamic factors are identified which allow identification of the term structure and its behaviour over a period of several years: the long-term level, the slope or difference between the long and short term, and the curvature or transition from the short to the long term. The panels in the left-hand column separate the component common to the whole euro area from the country-specific components, while the panels in the right-hand column represent the significant level, slope and curvature for each country as an aggregation of their common and specific components.

be considerable at certain times. As a result, the shorter-term inflation compensation differs more between euro area countries than that at longer terms.

Chart 3 illustrates this breakdown for inflation compensation on swaps at different time horizons: for expected average inflation in one year's time, in 10 years' time, between four 
1 ONE-YEAR COMPENSATION

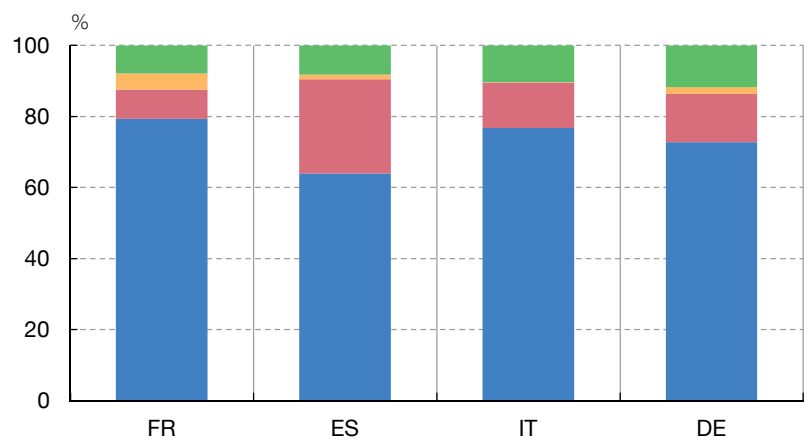

3 FIVE-YEAR COMPENSATION FIVE YEARS AHEAD

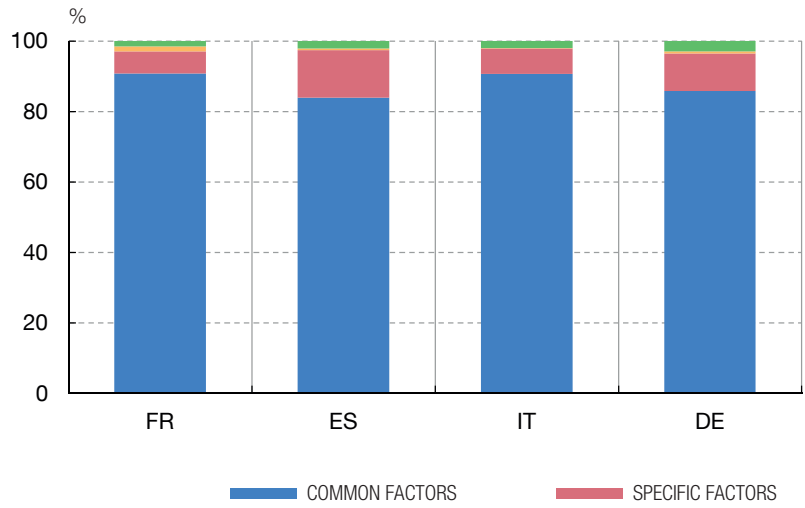

2 TEN-YEAR COMPENSATION

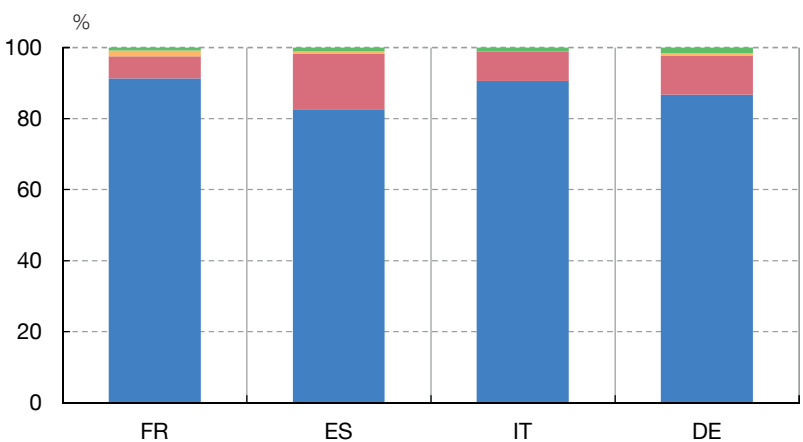

4 ONE-YEAR COMPENSATION FOUR YEARS AHEAD

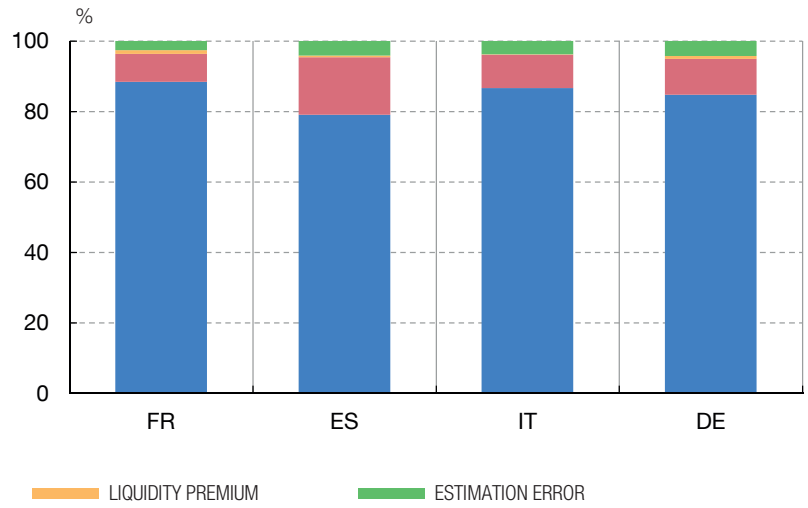

SOURCE: Banco de España.

a Breakdown obtained using the model of Gimeno and Ortega (2016).

Recent behaviour of inflation expectations and five years' time (one-year forward four years ahead), and between five and ten years' time (five-year forward five years ahead). It shows the average breakdown throughout the estimation period, from 2004 to the present. It is plain that the common component to the whole euro area is that which explains in the greatest proportion the inflation component compensation at all time horizons. The proportion explained by the country-specific component decreases with increasing term and it differs across countries, being higher in Spain and lower in France.

As noted in Section 2, the inflation compensation under swap contracts includes both expected average inflation for the relevant term and any possible risk premium. To speak of expectations in the strict sense of the term, these two variables would have to be separated. The estimated model can be used to decompose this inflation compensation into expectations and risk premium. To do this, it suffices to assume that the inflation compensation figure at the shortest term (one year) does not contain any risk premium associated with uncertainty as to the future, and to use the dynamic model to make predictions of how one-year expected inflation will behave in 10 years' time. These predictions would be the market expectations of inflation behaviour, ${ }^{9}$ and the difference between the inflation compensation on swaps and these expectations would be the risk premium. The top panels of Chart 4 show this breakdown on average for the

9 After deducting liquidity risk, which is estimated in the model. 
1 ONE-YEAR COMPENSATION FOUR YEARS AHEAD

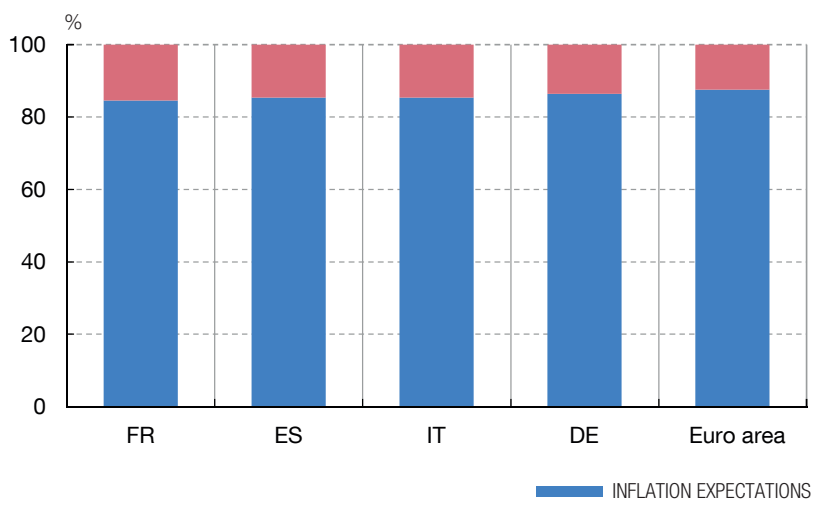

3 FIVE YEAR INFLATION RISK PREMIUM FIVE YEARS AHEAD

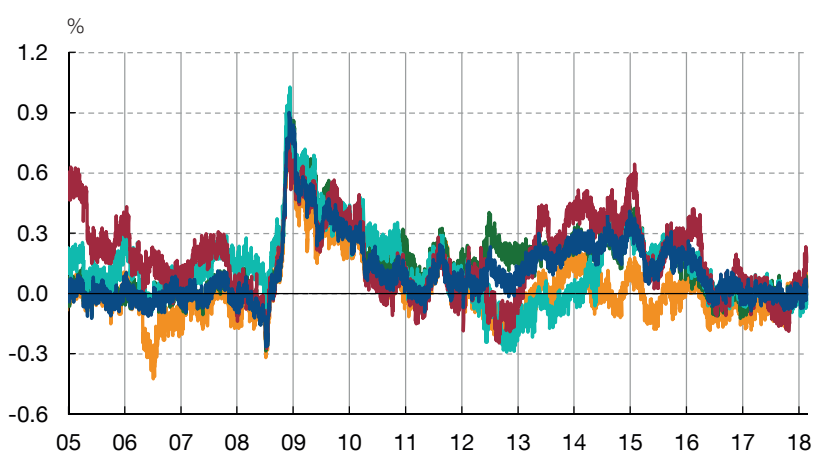

DE $\mathrm{DE} \longrightarrow \mathrm{IT}$ ES $\longrightarrow$ EURO AREA
2 FIVE-YEAR COMPENSATION FIVE YEARS AHEAD

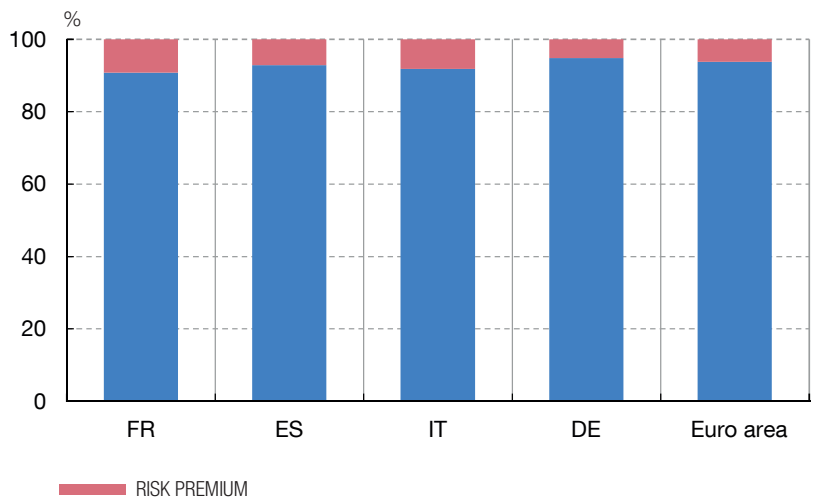

4 FIVE-YEAR COMPENSATION FIVE YEARS AHEAD

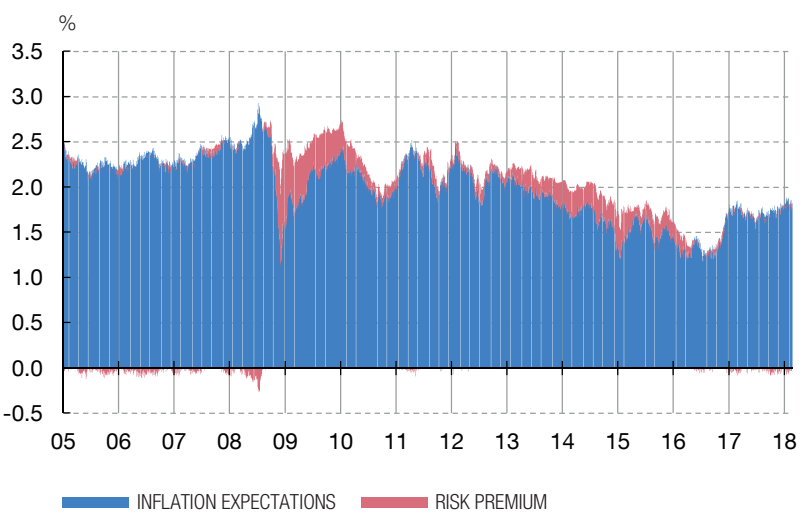

SOURCE: Banco de España.

a Breakdown obtained using the model of Gimeno and Ortega (2016).

whole period for expected inflation at medium-term time horizons, namely one-year forward four years ahead and five-year forward five years ahead, i.e. the expected average inflation between five and ten years from now. As can be seen, the inflation compensation implicit in the swaps is basically the inflation expectation at those medium-term time horizons; the premium takes very low values on average for all countries and for the total euro area.

The bottom panels of Chart 4 provide more information on the estimation of average inflation expectations for the 5-10 year time horizon. The bottom left-hand panel shows that the risk premium component associated with this time horizon is small although it has fluctuated over time. In the past two years it decreased considerably in all countries to become practically zero. The bottom right-hand panel shows how the estimated inflation expectations exhibited a downward trend from 2012, dropping to values near $1 \%$ at the beginning of 2015, just before the asset purchase programmes were extended to include sovereign bonds. Subsequently, the trend reversed, especially in the second half of 2016, possibly in response to the accumulation of unconventional monetary policy measures in the euro area from mid-2014. In 2017 the medium-term inflation expectations were below $2 \%$, at around $1.7 \%$ on average, still below those seen in the period before the economic crisis. 
DIEBOLD, F. X., C. LI and V. Z. YUE (2008). "Global Yield Curve Dynamics and Interactions: a Dynamic Nelson-Siegel Approach", Journal of Econometrics, 146(2), pp. 351-363.

DRAGHI, M. (2014). "Unemployment in the euro area", Annual central bank symposium in Jackson Hole, 22 August 2014.

- (2016). "How central banks meet the challenge of low inflation", Marjolin lecture, SUERF conference organised by the Deutsche Bundesbank, Frankfurt, 4 February 2016.

- (2017). "Accompanying the economic recovery", inaugural address of the ECB Forum on Central Banking, Sintra, 27 June 2017.

FUERTES, A. and R. GIMENO (2017). "Inflation expectation indicators based on financial instrument prices", Economic Bulletin 3/2017, Banco de España.

GIMENO, R. and A. IBÁÑEZ (2017). "The Eurozone (Expected) Inflation: An Option's Eyes View", Working Paper 1722, Banco de España.

GIMENO, R. and E. ORTEGA (2016). "The Evolution of Inflation Expectations in Euro Area Markets", Working Paper 1627, Banco de España.

NELSON, C. R. and A. F. SIEGEL (1987). "Parsimonious Modeling of Yield Curves", Journal of Business, 60(4), pp. 473-489. 